\title{
FOAM is the Concept: A Medicina no Século XXI?
}

\section{FOAM is the Concept: Medicine in the $21^{\text {st }}$ Century?}

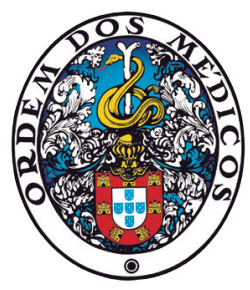

Mariana BAROSA $\triangle 1$, Pedro Caiado FERREIRA ${ }^{2}$, Bernardo PIMENTEL ${ }^{3}$, Ricardo MARQUES ${ }^{1}$

Acta Med Port 2021 Jul-Aug;34(7-8):495-497 - https://doi.org/10.20344/amp.14307

Palavras-chave: COVID-19; Educação Médica; Pandemia; Publicação em Acesso Aberto

Keywords: COVID-19; Education, Medical; Open Access Publishing; Pandemics

\section{INTRODUÇÃO}

A pandemia COVID-19 que atravessamos exigiu, inesperadamente, uma extraordinária mobilização de recursos e dinâmica à comunidade médica e científica. Ao desconhecimento inicial somou-se uma exponencial difusão de informação, que colocou enormes desafios aos processos de validação tradicionais e fez desde cedo denotar a importância do movimento internacional FOAM (Free Open Access Medical Education). Porventura desconhecido por muitos, FOAM faz parte de um movimento mais amplo designado 'Ciência Aberta' que tem crescido nos últimos anos. ${ }^{1}$ Este artigo procura dar a conhecer este novo conceito e mostrar como a comunidade FOAM tem sido crucial tanto no combate a esta crise como no estabelecimento de uma rede global de educação médica.

\section{Pertinência}

Enquanto autores 'juniores', em pleno início de um percurso formativo e com o entusiasmo que nos compete, sentimo-nos na obrigação de trazer ao domínio público aquilo que acreditamos que terá uma forte influência no futuro da medicina. Exercer a 'melhor' medicina centrada-na-pessoa com base na melhor ciência disponível é aquilo que nos move.

\section{Definição do conceito}

A Internet foi quiçá uma das grandes invenções do século passado. De facto, o mundo deixou-se moldar pelo progresso tecnológico e a medicina não foi excepção: pense-se nas vezes que utilizamos plataformas online de acesso a informação (PubMed, UpToDate, etc), de submissão de trabalhos ou e-learning. Cadogan, um emergencista australiano, teria isso presente quando cunhou o termo 'FOAM' em 2012, definindo-o como uma "combinação entre práticas altruístas e avanços tecnológicos com o objetivo de criar uma rede de educação médica gratuita e de livre acesso". ${ }^{2}$ A disseminação deste movimento permitiu que a Internet oferecesse mais que 'apenas' o abandono do papel e a correspondência por correio electrónico. Com o adven- to da FOAM, muito sustentado pelas redes sociais, testemunhamos uma produção científica comunitária online em tempo-real.

Trata-se, portanto, de uma comunidade que vive de websites, blogs, podcasts, etc, e se encontra virtualmente, em especial no Twitter. ${ }^{3}$ Esta é uma plataforma prática para difusão e discussão de conteúdo. Vive-se um autêntico espírito de partilha de conhecimento entre autores e leitores, mas também se fomenta: 1) a abertura ao desconhecido e a troca de incertezas e dúvidas que caracterizam o dia-a-dia do médico; 2) um espírito crítico, como nos mostra a rubrica mensal Things We Do For No Reason do Journal of Hospital Medicine.

Assim, FOAM é, acima de tudo, uma filosofia (um ethos). ${ }^{4}$ Incentiva o livre acesso à educação médica de alta qualidade para que, em qualquer parte do mundo, se possa exercer a melhor medicina à luz do conhecimento mais atual.

\section{FOAM no mundo e em Portugal}

Volvidos oito anos do seu nascimento, um crescente número de plataformas e hashtags '\#FOAMed' inundou o ciberespaço internacional. Objetivamente, a maioria dos seus utilizadores está ainda concentrada em poucos países, como os EUA, Canadá, Reino Unido e Austrália. Contudo, o acesso à Internet permitiu a disseminação deste conceito, inclusive para países menos desenvolvidos. ${ }^{5}$

Muitos médicos portugueses já terão consultado um dos vários exemplos listados na Tabela 1 sem conhecer a dimensão que os une. FOAM propaga-se fundamentalmente através de redes informais de comunicação e networking. Em Portugal não conhecemos exemplos explícitos de FOAM, mas dois sites que têm merecido maior destaque durante a pandemia - Evidentia Médica e MGFamiliar - aproximam-se do conceito: difundem conteúdo formativo gratuitamente em diversos formatos e os seus autores participam ativamente nas redes sociais.

1. Serviço de Medicina 4. Hospital Professor Doutor Fernando da Fonseca. Amadora. Portugal.

2. Serviço de Medicina 2.3. Hospital Santo António dos Capuchos. Lisboa. Portugal.

3. Serviço de Medicina Interna. Hospital da Luz. Lisboa. Portugal.

$\triangle$ Autor correspondente: Mariana Barosa. mmbarosa@gmail.com

Recebido: 10 de junho de 2020 - Aceite: 03 de dezembro de 2020 - First published: 05 de janeiro de 2021 - Online issue published: 01 de julho de 2021 Copyright @ Ordem dos Médicos 2021 
Tabela 1 - Exemplos de recursos de livre acesso recomendados pelos autores

\begin{tabular}{|c|c|}
\hline Medicina de Emergência & $\begin{array}{l}\text { EMCrit* - blog, podcast - emcrit.org } \\
\text { First10EM* - blog - first10em.com } \\
\text { SGEM - Skeptics Guide to Emergency Medicine* - blog - thesgem.com } \\
\text { Rebel EM* - blog - rebelem.com } \\
\text { Core EM* - blog, podcast - coreem.net } \\
\text { FOAMCast* - podcast - foamcast.org } \\
\text { Emergency Medicine Cases* - blog, podcast - emergencymedicinecases.com } \\
\text { The Bottom Line* - blog - thebottomline.org.uk }\end{array}$ \\
\hline Medicina Intensiva & $\begin{array}{l}\text { Internet Book of Critical Care }{ }^{*} \text { - blog, podcast - emcrit.org/ibcc/toc } \\
\text { Thinking Critical Care* - blog - thinkingcriticalcare.com }\end{array}$ \\
\hline Medicina Interna & $\begin{array}{l}\text { Internal Medicine Curbsiders* } \text { - podcast - thecurbsiders.com } \\
\text { Core IM* - podcast - coreimpodcast.com } \\
\text { Clinical Problem Solvers* - site, podcast - clinicalproblemsolving.com } \\
\text { The Curious Clinician - podcast - curiousclinicians.com } \\
\text { MedEd Pittsburgh* - página de Twitter - twitter.com/MedEdPGH }\end{array}$ \\
\hline Medicina Geral e Familiar & Best Science Podcast - podcast - therapeuticseducation.org \\
\hline Pediatria & Cribsiders* - podcast - thecurbsiders.com/thecribsiders \\
\hline Cardiologia & $\begin{array}{l}\text { This week in cardiology* - podcast - medscape.com/index/list_10295_0 } \\
\text { CardioNerds* - site, podcast - cardionerds.com }\end{array}$ \\
\hline Nefrologia & $\begin{array}{l}\text { NephJC* - journal club, blog, podcast - nephjc.com } \\
\text { NephMadness* - blog - ajkdblog.org/category/nephmadness } \\
\text { Precious Bodily Fluids* - blog - pbfluids.com }\end{array}$ \\
\hline Hemato-oncologia & Plenary Session - podcast - vinayakkprasad.com/plenarysession \\
\hline Dermatologia & DermNet NZ* - site - dermnetnz.org \\
\hline Oftalmologia & Ophtobook - livro - timroot.com/ophthobook \\
\hline Radiologia & Radiopaedia* - site - radiopaedia.org \\
\hline Ecografia de cabeceira & The Ultrasound Podcast* - site, podcast - coreultrasound.com \\
\hline Electrocardiografia & $\begin{array}{l}\text { Dr. Smith's ECG Blog* - blog - hqmeded-ecg.blogspot.com } \\
\text { Life in the fast lane* - blog - litfl.com }\end{array}$ \\
\hline
\end{tabular}

* recursos explicitamente FOAM

\section{As potencialidades FOAM}

\section{i) Revisão por pares pós-publicação}

Em 2006, Richard Smith - ex-editor-chefe do British Medical Journal (BMJ) e ex-CEO do BMJ Publishing Group -, num extenso artigo sobre revisão por pares pré-publicação, apontava as fragilidades e ineficiências desta tipologia de revisão. ${ }^{6}$ Levantava a hipótese de uma ativa revisão pós-publicação, transparente e aberta a todos, poder melhorar a qualidade da ciência publicada. FOAM utiliza este modelo mais dinâmico, tanto revendo investigação original publicada em revistas científicas, como revendo o próprio conteúdo FOAM, podendo ser um princípio de resposta às lacunas apontadas por Smith. Promove uma discussão comunitária ativa sobre as mais recentes publicações, envolvendo com frequência autoridades científicas reconhecidas. Este debate transparente e democrático, em plataformas de livre acesso como o Twitter à medida que ensaios clínicos vão sendo publicados, foi especialmente notório na resposta à COVID-19. Veja-se a onda crítica gerada na sequência do estudo de Mehra et al sobre o uso de hidroxicloroquina, que culminou na carta aberta escrita por mais de 100 cientistas internacionais aos editores da Lancet manifestando a sua preocupação quanto à integridade dos dados. ${ }^{7}$ Face à celeuma gerada, em poucos dias a Lancet e Mehra et al reagiram, e o artigo original foi retirado após nova revisão por pares solicitada pelos próprios autores. ${ }^{8}$

\section{ii) Translação de conhecimento}

Um dos problemas da translação do progresso científico para benefício da saúde populacional é a sua ampla latência temporal, que pode durar até 17 anos. ${ }^{9}$ FOAM poderá ter a vantagem de a encurtar, ${ }^{4}$ como se verificou nesta pandemia. Vários foram os exemplos FOAM que rapidamente dissecaram a informação disponível e a divulgaram à comunidade médica da linha da frente. Notável foi o exemplo do livro online em permanente construção Internet Book of Critical Care, que, a 2 de março de 2020, criou um capítulo dedicado à COVID-19, quando ainda só tinham sido identificados dois casos em Portugal (à data em que escrevemos já tem mais de 100 citações e 200 comentários). Também desde essa altura, o Evidentia Médica tem desenvolvido um impressionante repositório dos melhores artigos publicados sobre a COVID-19. 


\section{Limitações}

Em primeiro lugar, FOAM não se define como método de investigação e, portanto, não gera prova científica. É antes uma 'digestão comunitária' dos produtos dessa mesma investigação, com as limitações que lhe são inerentes. Em segundo, critica-se frequentemente a falta de controlo de qualidade dos conteúdos e competência científica dos autores. ${ }^{4,10}$ FOAM assenta em meios de rápida difusão de informação que pode ser perigosa e com consequências desproporcionais aos benefícios. Tal implica que seja utilizada judiciosamente e tendo em conta as particularidades e imperfeições dos seus métodos de validação. Na sua versão menos escolástica, o Twitter, FOAM poderá equivaler a uma conversa de corredor, mas temas mais complexos não devem ser simplificados em 'meia-dúzia de caracteres'. Em terceiro, na medida em que blogs e podcasts dão espaço à visão do autor, FOAM acarreta alguma personalização dos conteúdos, o que, aliado à maior proximidade entre autores e leitores, aumenta o risco de se regressar a uma medicina 'baseada na eminência'. Além disso, é importante não descurar a fiscalização de imperativos éticos como conflitos de interesse e confidencialidade dos dados clínicos. Por fim, a cobertura de outras áreas para além do doente

\section{REFERÊNCIAS}

1. Donato H, Villanueva T, Escada P. Publicação Médica em Tempo de Pandemia. Rev Soc Port Med Intern. 2020; Publicação Especial COVID-19:42.

2. Shaw G. Don't call it social media: FOAM and the future of medical education. Emerg Med News. 2013;35.2:1-30.

3. Jalali N, Carter C, Alfaraj S, Moghtaderi A, Pines J. Trends and Predictors of Retweets in Free Open Access Medical Education (\#FOAMed) on Twitter (2013-2017). Acad Emerg Med. 2019;26:443-6.

4. Nickson CP, Cadogan MD. Free Open Access Medical education (FOAM) for the emergency physician. Emerg Med Australas. 2014;26:76-83.

5. Burkholder TW, Bellows JW, King RA. Free open access medical education (FOAM) in emergency medicine: the global distribution of users in 2016. West J Emerg Med. 2018;19:600.

6. Smith R. Peer review: a flawed process at the heart of science and crítico encontra-se ainda numa fase muito incipiente.

Conhecer estas limitações é essencial para usar FOAM de forma responsável. ${ }^{10}$

\section{CONCLUSÃO}

Acreditamos que esta pandemia tornou evidente que acompanhar sozinho todo o novo conhecimento científico é humanamente impossível. Afortunadamente, o ser humano prospera em comunidade e floresce na entreajuda. Perante a vertiginosa propagação de notícias relativamente à $\mathrm{CO}$ VID-19, acreditamos que a rapidez, abertura e transparência da comunidade FOAM têm sido muito importantes na gestão desta crise sanitária global. Será FOAM o 'conceito' para a medicina no século XXI?

\section{CONTRIBUTO DOS AUTORES}

MB, PCF, BP: Concepção do artigo.

RM: Revisão do artigo.

\section{CONFLITOS DE INTERESSE}

Os autores declaram não ter conflitos de interesse relacionados com o presente trabalho.

7. Watson J. An open letter to Mehra et al and The Lancet; 2020. [consultado 2020 Jun 3]. Disponível em: https://zenodo.org/record/3864691\#. XtgJBi90o1K.

8. Mehra MR, Ruschitzka F, Patel AN. Retraction-Hydroxychloroquine or chloroquine with or without a macrolide for treatment of COVID-19: a multinational registry analysis. Lancet. 2020;395(10240):1820.

9. Morris ZS, Wooding S, Grant J. The answer is 17 years, what is the question: understanding time lags in translational research. JRSM Open. 2011;104:510-20.

10. Allen NG, Chandrasekaran EB, Goett RR, Kluesner KH, Vearrier L. We Must Analyze and Clear Up the Ethical Issues in FOAM. ACEP Now. 2018;37:11. 